TRANSACTIONS OF THE

AMERICAN MATHEMATICAL SOCIETY

Volume 352, Number 7, Pages 3305-3322

S 0002-9947(00)02488-0

Article electronically published on March 24, 2000

\title{
SHIFT EQUIVALENCE AND THE CONLEY INDEX
}

\author{
JOHN FRANKS AND DAVID RICHESON
}

\begin{abstract}
In this paper we introduce filtration pairs for an isolated invariant set of continuous maps. We prove the existence of filtration pairs and show that, up to shift equivalence, the induced map on the corresponding pointed space is an invariant of the isolated invariant set. Moreover, the maps defining the shift equivalence can be chosen canonically. Last, we define partially ordered Morse decompositions and prove the existence of Morse set filtrations for such decompositions.
\end{abstract}

\section{INTRODUCTION}

One would like to study the topological properties of an isolated invariant set $S$ for a continuous map $f: X \rightarrow X$. If $f$ is, for instance, an Axiom A diffeomorphism with the no-cycle property and $S$ is a basic set, then one may isolate $S$ between levels of a filtration and look at the relative homology of these sets. In general, $S$ does not arise this way. The discrete Conley index is a tool used to study precisely such sets. The ideas of Conley $\mathrm{C}$ were developed by many people using, for instance, shape theory [RS], algebraic topology $[\mathrm{Mr}$ and category theory $[\mathrm{Sz}]$. Due to the daunting nature of these subjects many casual readers find Conley index theory inaccessible. In this paper we try to develop Conley index theory in an intuitive fashion relying on no advanced mathematics.

We begin by introducing the filtration pair. This topological pair has many of the benefits of an actual filtration while possessing the versatility of an index pair (the standard object of study in Conley index theory). To each filtration pair $P=(N, L)$ we may associate a pointed topological space and an induced map $f_{P}: N_{L} \rightarrow N_{L}$. This space itself is not independent of the choice of filtration pair, but its shift equivalence class is. Shift equivalence is a natural equivalence relation for non-invertible dynamical systems which is related to topological conjugacy, but is less rigid. It was introduced by R. Williams for (among other things) the study of low dimensional hyperbolic invariant sets (see [W] for more details and references). We call the shift equivalence class, in the homotopy category, of the map on the pointed space the discrete homotopy Conley index of $S$.

This construction has several nice properties, not the least of which is its relative simplicity. We see that the basepoint is a super-attractor; that is, $f_{P}^{-1}(*)$ is a neighborhood of the basepoint $*$. Also, unlike index pairs, filtration pairs are robust under small $C^{0}$ perturbations of $f$. If $X$ is a manifold, then $(N, L)$ may be chosen to be homeomorphic to a finite simplicial pair. Thus, the (co)homology of the filtration pair is necessarily finite dimensional. Last, having the homotopy index

Received by the editors June 22, 1998 .

2000 Mathematics Subject Classification. Primary 37B30.

(C)2000 American Mathematical Society 
one may apply various functors to it such as homology, cohomology, $\pi_{n}$, the Leray functor, direct limits, etc., thus obtaining the Conley indices found in the literature.

Next, we investigate decompositions of isolated invariant sets. We define attractors and repellers as a motivation so that we may define partially ordered Morse decompositions. We show that Morse set filtrations exist for such decompositions.

Finally, we discuss Szymczak's categorical definition of the Conley index. We show that, in fact, our construction contains exactly the same information as his construction.

\section{ISOLATED INVARIANT SETS}

Let $U$ be an open subset of a locally compact metric space $X$ and suppose $f: U \rightarrow X$ is a continuous map.

Definition 2.1. For any set $N \subset U$ define $\operatorname{Inv}^{m} N$ to be the set of $x \in N$ such that there exists an orbit segment $\left\{x_{n}\right\}_{-m}^{m} \subset N$ with $x_{0}=x$ and $f\left(x_{n}\right)=x_{n+1}$ for $n=-m, \ldots, m-1$. We will call a complete orbit containing $x$ a solution through $x$. More precisely, if $\sigma: \mathbb{Z} \rightarrow N$ is given by $\sigma(n)=x_{n}$ and $x_{0}=x$ and $f\left(x_{n}\right)=x_{n+1}$ for all $n$, we will call $\sigma$ a solution through $x$. We define the maximal invariant subset, Inv $N$ to be $\operatorname{Inv}^{\infty} N$, the set of $x \in N$ such that there exists a solution $\sigma$ with $\{\sigma(n)\}_{-\infty}^{\infty} \subset N$ and with $\sigma(0)=x$.

Note that from the definition it is clear that $f(\operatorname{Inv} N)=\operatorname{Inv} N$. The following basic property of Inv $N$ is trivial if $f$ is one-to-one, but requires proof in the case of a general continuous map.

Proposition 2.2. If $U$ is an open subset of a locally compact metric space $X, f$ : $U \rightarrow X$ is continuous and $N$ is a compact subset of $U$, then

$$
\operatorname{Inv} N=\bigcap_{m=0}^{\infty} \operatorname{Inv}^{m} N
$$

Proof. Trivially Inv $N \subset \bigcap_{m=0}^{\infty} \operatorname{Inv}^{m} N$ so we need only prove the reverse inclusion. If $x \in \bigcap_{m=0}^{\infty} \operatorname{Inv}^{m} N$, then we must show that $x \in \operatorname{Inv} N$.

Let $X_{1}=f^{-1}(x) \cap N$ and, for $k>1$, inductively define $X_{k}=f^{-1}\left(X_{k-1}\right) \cap N$ so each $X_{k}$ is non-empty, compact and $f\left(X_{k}\right) \subset X_{k-1}$. Then define

$$
Y_{k}=\bigcap_{n \geq 1} f^{n}\left(X_{n+k}\right)
$$

Since $\left\{f^{n}\left(X_{n+k}\right)\right\}$ is a nested sequence of non-empty compact subsets of $X_{k}$, it follows that $Y_{k}$ is a non-empty compact subset of $X_{k}$. So $f\left(Y_{1}\right)=\{x\}$ and for $k>1$,

$$
f\left(Y_{k}\right)=\bigcap_{n \geq 1} f^{n+1}\left(X_{n+k}\right)=\bigcap_{n \geq 2} f^{n}\left(X_{n+k-1}\right)=Y_{k-1}
$$

Thus every point of $Y_{k-1}$ has an $f$ pre-image in $Y_{k}$. Define $x_{-1}$ to be any point of $Y_{1}$ (so $\left.f\left(x_{-1}\right)=x\right)$ and inductively define $x_{-k}$ to be a point in $Y_{k}$ such that $f\left(x_{-k}\right)=x_{-k+1}$. If we also define $x_{k}=f^{k}(x)$ for $k \geq 0$, then $\sigma: \mathbb{Z} \rightarrow N$, given by $\sigma(n)=x_{n}$, is a solution through $x$. It follows that $x \in \operatorname{Inv} N$. 
Definition 2.3. A compact set $N$ is called an isolating neighborhood 1 if Inv $N \subset$ Int $N$. A set $S$ is called an isolated invariant set if there exists an isolating neighborhood $N$ with $S=\operatorname{Inv} N$. A compact set $N$ is called an isolating block if $f(N) \cap N \cap f^{-1}(N) \subset \operatorname{Int} N$.

Notice that every isolating block $N$ is an isolating neighborhood for the set $S=\operatorname{Inv} N$. The converse is not true.

Suppose $N$ is an isolating neighborhood.

Definition 2.4. We define the exit set of $N$ to be

$$
N^{-}:=\{x \in N: f(x) \notin \operatorname{Int} N\} .
$$

That is, $N^{-}$is the subset consisting of those points in $N$ whose image is in the complement of the interior of $N$.

\section{Filtration PAirs}

Ideally, for the study of an isolated invariant set $S$ we would like a pair $(N, L)$ of spaces forming part of a filtration; i.e. we would like to have $L \subset N$ compact, $S=\operatorname{Inv}(N \backslash L), f(L) \subset \operatorname{Int} L$, and $f(N) \subset \operatorname{Int} N$. This is much too strong, however, as we often need to study $f$ on a small neighborhood of $S$ and there may not exist a forward invariant small neighborhood $N$ of $S$.

To motivate the proper modification of the notion of a filtration pair, we will look at the definition of a filtration. Consider a map $f$ defined on a neighborhood of a pair of compact sets $(N, L)$ equal to the closures of their interiors with $f(N) \subset N$ and $f(L) \subset L$. Then the condition $f(L) \subset \operatorname{Int} L$ is equivalent to $f(L) \cap \operatorname{cl}(N \backslash L)=$ $\emptyset$. Also, given this, the condition $f(N) \subset \operatorname{Int} N$ is equivalent to $f(\operatorname{cl}(N \backslash L)) \subset \operatorname{Int} N$. We take these two conditions for the case when $N$ and $L$ are not forward invariant. Notice that $f(\operatorname{cl}(N \backslash L)) \subset \operatorname{Int} N$ is equivalent to requiring that $L$ be a neighborhood of $N^{-}$. This requirement also asserts that any point of $N$ which is mapped by $f$ outside of $N$ must be in $L$. Thus we have the following definition.

Definition 3.1. Let $S$ be an isolated invariant set and suppose $L \subset N$ is a compact pair contained in the interior of the domain of $f$. The pair $(N, L)$ is called a filtration pair for $S$ provided $N$ and $L$ are each the closures of their interiors and

1. $\operatorname{cl}(N \backslash L)$ is an isolating neighborhood of $S$,

2. $L$ is a neighborhood of $N^{-}$in $N$, and

3. $f(L) \cap \operatorname{cl}(N \backslash L)=\emptyset$.

The definition of filtration pair here is close to, but somewhat more general than, the concept of filtration pair introduced in $[\overline{B F}$, where the intent was to study dynamical systems satisfying stronger hypotheses than we now wish to assume. Also, our definition of filtration pair is close to, but somewhat less general than, the definition of index pair introduced by Conley and studied by several authors (see $[\underline{\mathrm{E}}], \underline{\mathrm{Mr}}]$, and $\underline{\mathrm{Sz}}]$ ). It is significantly less general than the definition of index pair found in [RS].

\footnotetext{
${ }^{1}$ This definition of isolating neighborhood differs slightly from that used by R. Easton in $[\mathrm{E}$, for example. There, $N$ is called an isolating neighborhood provided $\bigcap_{m \in \mathbb{Z}} f^{m}(N)$ is in the interior of $N$. For a map which is not one-to-one the sets $\operatorname{Inv} N$ and $\bigcap_{m \in \mathbb{Z}} f^{m}(N)$ may not coincide, as is pointed out in $[\mathrm{E}$.
} 
We have another property that is analogous to one for a filtration. If $\left(N_{1}, L\right)$ and $\left(N_{2}, L\right)$ are filtration pairs for isolated invariant sets $\operatorname{Inv}\left(N_{1} \backslash L\right)$ and $\operatorname{Inv}\left(N_{2} \backslash L\right)$ and $N_{1} \subset \operatorname{Int} N_{2}$, then $\left(N_{2}, N_{1}\right)$ is a filtration pair for $\operatorname{Inv}\left(N_{2} \backslash N_{1}\right)$.

Suppose $f: X \rightarrow X$ is a continuous map and $x \in X$. We define the $\omega$-limit set of $x$ to be

$$
\omega(x)=\bigcap_{N>0} \operatorname{cl}\left(\bigcup_{n>N} f^{n}(x)\right) .
$$

We will need the following easy proposition in our study of filtration pairs.

Proposition 3.2. If $P=(N, L)$ is a filtration pair for $S$ and $x \in N$ satisfies $f^{n}(x) \in N \backslash L$ for all $n>0$, then $\omega(x) \subset S$.

Proof. If $n_{i} \rightarrow \infty$ and the sequence $\left\{f^{n_{i}}(x)\right\}$ converges to $z \in \operatorname{cl}(N \backslash L)$, then for any $k \in \mathbb{Z}$ the sequence $\left\{f^{n_{i}+k}(x)\right\}$ is well defined for $i$ sufficiently large and converges to a point $z_{k} \in \operatorname{cl}(N \backslash L)$. By continuity of $f$, if $k>0$, then $z_{k}=f^{k}(z)$ and if $k<0$, then $z \in f^{-k}\left(z_{k}\right)$. It follows that $z \in \operatorname{Inv} \operatorname{cl}(N \backslash L)=S$.

Our next objective is to prove the existence of isolating blocks and filtration pairs inside any neighborhood of $S$.

Recall that for any $\varepsilon>0$, a sequence $\left\{x_{n}\right\}_{q}^{p}$ is called an $\varepsilon$-chain provided

$$
d\left(f\left(x_{n}\right), x_{n+1}\right)<\varepsilon
$$

for all $n=q, \ldots, p-1$.

Definition 3.3. For any isolating neighborhood $N$ of an isolated invariant set $S$ and any $\varepsilon>0$ define the $\varepsilon$-chain neighborhood of $S$ relative to $N$ to be the set $C_{\varepsilon}(N, S)$ of all $x \in N$ such that, for some $k$, there exists an $\varepsilon$-chain $\left\{x_{n}\right\}_{-k}^{k} \subset N$ with $x=x_{0}$ and $x_{k}, x_{-k} \in S$.

In the next proposition we show that the sets $C_{\varepsilon}(N, S)$ for different $\varepsilon>0$ provide a neighborhood basis for $S$.

Proposition 3.4. Let $S$ be an isolated invariant set with isolating neighborhood $N$. For any neighborhood $V$ of $S$ in $N$ there is an $\varepsilon>0$ with $C_{\varepsilon}(N, S) \subset V$.

Proof. We may assume $V$ is open. From Proposition 2.2 it follows that there is an $m>0$ with $\operatorname{Inv}^{m} N \subset V$. Our proof is by contradiction. Suppose there is no $\varepsilon>0$ with $C_{\varepsilon}(N, S) \subset V$. Then for every $\varepsilon>0$ there is an $x(\varepsilon) \in C_{\varepsilon}(N, S) \backslash V$, i.e. there is an $\varepsilon$-chain $\left\{x_{n}(\varepsilon)\right\}_{-k(\varepsilon)}^{k(\varepsilon)} \subset N$ with $x(\varepsilon)=x_{0}(\varepsilon)$ and $x_{k(\varepsilon)}(\varepsilon), x_{-k(\varepsilon)}(\varepsilon) \in S$. Truncating these when $k(\varepsilon)>m$ or extending them to be orbit segments if $k(\varepsilon)<m$ we produce for each $\varepsilon>0$ a $\varepsilon$-chain $\left\{x_{n}(\varepsilon)\right\}_{-m}^{m} \subset N$ with $x_{0}(\varepsilon) \notin V$. Choosing a sequence $\varepsilon_{i}$ converging to 0 as $i \rightarrow \infty$, we may assume that each of the sequences $\left\{x_{j}\left(\varepsilon_{i}\right)\right\},-m \leq j \leq m$ has a limit, say $x_{j}$. Clearly $x_{j} \in N$ and $x_{0} \in N \backslash V$. Also it is clear by continuity of $f$ that $f\left(x_{j}\right)=x_{j+1}$ for $-m \leq j<m$. Hence $x_{0} \in \operatorname{Inv}^{m} N \subset V$ which is a contradiction.

We now give a proof of a result due to R. Easton $[\mathrm{E}]$ which asserts the existence of isolating blocks. We essentially reproduce Easton's proof, because it is short, elementary and quite elegant.

Proposition 3.5. Every neighborhood of an isolated invariant set $S$ contains an isolating block. In particular if $N$ is an isolating neighborhood for $S$, then for every sufficiently small $\varepsilon>0, \operatorname{cl}\left(C_{\varepsilon}(N, S)\right)$ is an isolating block. 
Proof. Let $B=\operatorname{cl}\left(C_{\varepsilon}(N, S)\right)$. If $B$ is not an isolating block for $S$, then there is a point $x$ such that $x, f(x)$ and $f^{2}(x)$ are all in $B$, but $f(x) \notin \operatorname{Int} B$. By continuity of $f$ there exists $\delta>0$ such that if $d(x, y)<\delta$, then $d(f(x), f(y))<\varepsilon$. Since $x \in B$ and $f^{2}(x) \in B$ there are $\varepsilon$-chains $\left\{x_{n}\right\}_{0}^{k}$ with $x_{0} \in S$ and $d\left(x_{k}, x\right)<\delta$, and $\left\{y_{n}\right\}_{0}^{m}$ with $y_{m} \in S$ and $d\left(y_{0}, f^{2}(x)\right)<\varepsilon$. Then the sequence $x_{0}, x_{1}, \ldots, x_{k}, f(x), y_{0}, \ldots, y_{m}$ is an $\varepsilon$-chain from $S$ to $S$ containing $f(x)$. This implies $f(x) \in C_{\varepsilon}(N, S)=\operatorname{Int} B$, which is a contradiction.

Theorem 3.6. If $N$ is an isolating block and $L$ is any sufficiently small compact neighborhood of $N^{-}$in $N$, then $(N, L)$ is a filtration pair. Moreover there is a neighborhood of $f$ in the $C^{0}$ topology such that for any $\tilde{f}$ in this neighborhood, $\tilde{S}=\operatorname{Inv}(N \backslash L, \tilde{f})$ is an isolated invariant set and $(N, L)$ is a filtration pair for $\tilde{S}$.

Proof. To check property (1) of Definition 3.1 it suffices to show $S \subset N \backslash L$. Clearly $f\left(N^{-}\right)$is in the complement of Int $N$ and hence $S \cap f\left(N^{-}\right)=\emptyset$. Thus if $L$ is a sufficiently small neighborhood of $N^{-}$, we have $S \cap f(L)=\emptyset$. Since $S$ is invariant $S \cap L$ could only be non-empty if $S \cap f(L)$ were. Hence $S \cap L=\emptyset$ and $S \subset N \backslash L$.

Property (2) of Definition 3.1 is trivially satisfied. To check property (3) note first that since

$$
N \backslash N^{-} \subset N \cap f^{-1}(\operatorname{Int} N) \subset N \cap f^{-1}(N),
$$

we have $\operatorname{cl}\left(N \backslash N^{-}\right) \subset N \cap f^{-1}(N)$.

Also $f\left(N^{-}\right) \subset f(N) \cap(\operatorname{Int} N)^{c}$ where the superscript $c$ indicates complement. It follows that

$$
f\left(N^{-}\right) \cap \operatorname{cl}\left(N \backslash N^{-}\right) \subset\left(f(N) \cap(\operatorname{Int} N)^{c}\right) \cap N \cap f^{-1}(N)=\emptyset,
$$

because $f(N) \cap N \cap f^{-1}(N) \subset \operatorname{Int} N$.

Since $f\left(N^{-}\right)$and $\operatorname{cl}\left(N \backslash N^{-}\right)$are disjoint it is clear that if $L$ is a sufficiently small neighborhood of $N^{-}$, then $f(L)$ and $\operatorname{cl}(N \backslash L)$ are also disjoint. Thus property (3) of Definition 3.1 is satisfied.

Finally, we check the fact that $(N, L)$ is a filtration pair for maps which are $C^{0}$ close to $f$. First we note that $N$ is an isolating block for nearby maps. If this were not the case, then there would exist a sequence $\left\{f_{n}\right\}$ converging to $f$ and a sequence $\left\{x_{n}\right\}$ with $x_{n} \in f_{n}^{-1}(N) \cap N \cap f_{n}(N) \cap(\operatorname{Int} N)^{c}$. Choosing a subsequence we can assume $\left\{x_{n}\right\}$ converges to $z \in f^{-1}(N) \cap N \cap f(N) \cap(\operatorname{Int} N)^{c}$ which would contradict the fact that $N$ is an isolating block for $f$.

Similarly if $N_{n}^{-}=\left\{x \in N: f_{n}(x) \notin \operatorname{Int} N\right\}$ and $x_{n} \in N_{n}^{-} \cap(\operatorname{Int} L)^{c}$, then a subsequence of $\left\{x_{n}\right\}$ will converge to a point $z \in N^{-} \cap(\operatorname{Int} L)^{c}$ contradicting the hypothesis that $L$ is a neighborhood of $N^{-}$. Thus $(N, L)$ is a filtration pair for any map sufficiently $C^{0}$ close to $f$.

In [RS] Robbin and Salamon give a robustness theorem for index pairs (Theorem 5.3). We remarked following Definition 3.1 that they had a very general definition of index pairs. It should be noted that the robust index pairs constructed in the proof of this theorem are not filtration pairs nor are they index pairs in the sense this term is used in $\underline{\mathrm{E}}, \underline{\mathrm{Mr}}$, and $\underline{\underline{\mathrm{Sz}}}]$.

Theorem 3.7. Let $U$ be an open subset of an $n$-dimensional manifold $M$ and suppose $f: U \rightarrow M$ is a continuous map with an isolated invariant set $S$. Inside 
any neighborhood of $S$ there exists a filtration pair $(N, L)$ such that $N$ is an $n$ dimensional manifold with boundary and $\partial L$ is an $(n-1)$-dimensional submanifold of $M$. In particular $(N, L)$ is homeomorphic to a finite simplicial pair.

Proof. We may assume the given neighborhood $V$ is open in $M$ and $\operatorname{cl}(V)$ is a compact subset of $M$. Let $N_{1}$ be an isolating block for $S$ inside $V$.

Let $\varphi: \operatorname{cl}(V) \rightarrow \mathbb{R}^{+}$be a smooth function which vanishes exactly on $N_{1}$. For any regular value $\delta>0 N=\varphi^{-1}([0, \delta])$ is a compact manifold with boundary. If we choose $\delta$ small enough, $N$ will be an isolating block for $S$.

Now, let $\psi: \operatorname{cl}(V) \rightarrow \mathbb{R}^{+}$be a smooth map which vanishes exactly on $N^{-}$. For any regular value $\eta>0$ the space $\psi^{-1}([0, \eta])$ is a manifold with boundary. If $\eta>0$ is small enough and $L_{0}:=\psi^{-1}([0, \eta])$, then $L_{0}$ is a small neighborhood of $N^{-}$. Modifying $L_{0}$ slightly we may assume that the boundary of $N$ and the boundary of $L_{0}$ intersect transversely. If we define $L:=N \cap L_{0}$ and $\eta$ was chosen sufficiently small, then it follows from Theorem 3.6 that $(N, L)$ is a filtration pair. Triangulating $N$ with $L$ as a subcomplex shows that $(N, L)$ is homeomorphic to a finite simplicial pair.

Theorem 3.8. Let $P=(N, L)$ be a filtration pair for $f$ and let $N_{L}$ denote the quotient space $N / L$ where the collapsed set $L$ is denoted $[L]$ and is taken as the base-point. Then $f$ induces a continuous base-point preserving map $f_{P}: N_{L} \rightarrow N_{L}$ with the property $[L] \subset$ Int $f_{P}^{-1}([L])$. This map will be called the pointed space map associated to $P$.

Note that in case $L$ is empty, then $N / L$ is defined to be the disjoint union of $N$ and the single point space $[L]:=[\emptyset]$.

Proof. Let $p: N \rightarrow N / L$ be the quotient map and define $f_{P}([L])=[L]$ and $f_{P}(x)=p(f(x))$ otherwise, where we have identified $N_{L} \backslash\{[L]\}$ with $N \backslash L$. By Definition $3.1 f(L)$ is disjoint from $\operatorname{cl}(N \backslash L)$. Hence if $K$ is a sufficiently small neighborhood of $L$ in $N$, then $f(K)$ is disjoint from $\operatorname{cl}(N \backslash L)$. Hence $f_{P}(x)=[L]$ for all $x \in p(K)$.

It is immediate that $f_{P}$ is continuous on $N_{L} \backslash\{[L]\}$ since it is the composition of continuous functions there. To check continuity at $[L]$ note that if $\left\{x_{n}\right\}$ is a sequence in $N_{L} \backslash\{[L]\}$ converging to $[L]$, then $\left\{p^{-1}\left(x_{n}\right)\right\}$ is eventually in $K$ so $\left\{f_{P}\left(x_{n}\right)\right\}$ is a sequence which is eventually constant and equal to $[L]$.

The pointed space map $f_{P}$ depends on more than the invariant set $S$. Indeed even its homotopy type and the homotopy type of $N_{L}$ depend on the choice of filtration pair $P=(N, L)$. Our next section is devoted to an important equivalence relation for which all choices of filtration pair will give equivalent associated pointed space maps.

\section{ShifT EQUIVALENCE}

Suppose $\mathcal{K}$ is a category. Let $X, X^{\prime}$ be objects in $\mathcal{K}$ and $f: X \rightarrow X, g: X^{\prime} \rightarrow X^{\prime}$ be endomorphisms. We say that $(X, f)$ and $\left(X^{\prime}, g\right)$ are shift equivalent, $f \sim_{s} g$, if there exist $m \in \mathbb{Z}^{+}, r: X \rightarrow X^{\prime}$ and $s: X^{\prime} \rightarrow X$ such that the diagrams
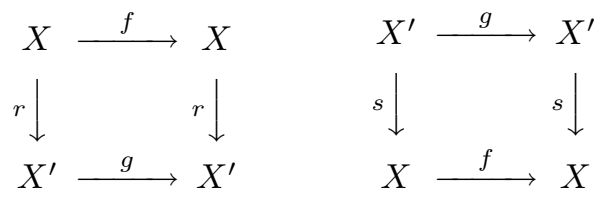
commute and $r \circ s=g^{m}$ and $s \circ r=f^{m}$. The integer $m$ is called the lag.

Shift equivalence is a natural and dynamically significant equivalence relation for maps. Note that if $f$ and $g$ are homeomorphisms (i.e. invertible), then they are shift equivalent if and only if they are topologically conjugate. If they are shift equivalent, a conjugacy is given by $h=r \circ f^{-m}=g^{-m} \circ r$ and $h^{-1}=s$.

We wish to prove that for any two filtration pairs of an isolated invariant set $S$ the corresponding maps on the pointed spaces are shift equivalent. We do this by first proving it for two special cases in the following lemmas.

Lemma 4.1. Suppose $P^{\prime}=\left(N, L^{\prime}\right)$ and $P=(N \cup L, L)$ are filtration pairs for $S$ and that $L^{\prime} \subset L$ and $f(L) \subset$ Int $L$. Then the induced maps, $f_{P^{\prime}}$ and $f_{P}$, on the corresponding pointed spaces are shift equivalent.

Proof. Let $Q=N \cup L$. There is a continuous function $r: N_{L^{\prime}} \rightarrow Q_{L}$ given by $r\left(\left[L^{\prime}\right]\right)=[L]$ and $r(x)=p_{2}(x)$ otherwise, where we have identified $N_{L^{\prime}} \backslash\left\{\left[L^{\prime}\right]\right\}$ with $N \backslash L^{\prime}$ and $p_{2}: Q \rightarrow Q_{L}$ is the quotient map. Clearly $r \circ f_{P}=f_{P^{\prime}} \circ r$.

Since $L^{\prime}$ contains the exit set of $N$ and $S$ is disjoint from $L$, there is an $n>0$ such that for every $x \in N \cap L$ we have $f^{k}(x) \in L^{\prime}$ for some $k<n$.

Define a map $s: Q_{L} \rightarrow N_{L^{\prime}}$ by $s([L])=\left[L^{\prime}\right]$ and $s(x)=f_{P^{\prime}}^{n}\left(p_{1}(x)\right)$ otherwise, where we have identified $Q_{L} \backslash\{[L]\}$ with $Q \backslash L=N \backslash L$ and $p_{1}: N \rightarrow N_{L^{\prime}}$ is the quotient map. Clearly $s \circ f_{P}=f_{P^{\prime}} \circ s$.

It is immediate that $s$ is continuous on $Q_{L} \backslash\{[L]\}$ since it is the composition of continuous functions there. To check continuity at $[L]$ note that there is a neighborhood $V$ of $L$ in $Q$ such that $f(V) \subset \operatorname{Int} L$ and hence for any point $z \in V$, we have $f^{k}(z) \in L^{\prime}$ for some $k \leq n$. Thus for any point $z \in N \cap V$, we have $f_{P^{\prime}}^{n}\left(p_{1}(z)\right)=\left[L^{\prime}\right]$. So $s(x)$ is constant on a neighborhood of $[L]$ in $Q_{L}$ and hence continuous at $[L]$.

It is also clear from the definitions that $s \circ r=f_{P^{\prime}}^{n}$ and $r \circ s=f_{P}^{n}$.

Lemma 4.2. Suppose $P=(N, L)$ and $P^{\prime}=\left(N^{\prime}, L\right)$ are filtration pairs for $S$ and that $N \backslash L \subset N^{\prime} \backslash L$ and $f(L) \subset \operatorname{Int} L$. Then the induced maps, $f_{P}$ and $f_{P^{\prime}}$, on the corresponding pointed spaces, are shift equivalent.

Proof. There is a continuous function $r: N_{L} \rightarrow N_{L}^{\prime}$ given by $r([L])=[L]$ and $r(x)=p^{\prime}(x)$ otherwise, where we have identified $N_{L} \backslash\{[L]\}$ with $N \backslash L$ and $p^{\prime}$ : $N^{\prime} \rightarrow N_{L}^{\prime}$ is the quotient map. Clearly $r \circ f_{P^{\prime}}=f_{P} \circ r$.

It follows from Proposition 3.2 that there is an $n>0$ such that $f^{n}\left(N^{\prime}\right) \subset \operatorname{Int} N$. Define $s: N_{L}^{\prime} \rightarrow N_{L}$ by $s([L])=[L]$ and $s(x)=p\left(f^{n}(x)\right)$ otherwise, where we have identified $N_{L}^{\prime} \backslash\{[L]\}$ with $N^{\prime} \backslash L$ and $p: N \rightarrow N_{L}$ is the quotient map. Clearly $s \circ f_{P^{\prime}}=f_{P} \circ s$.

It is immediate that $s$ is continuous on $N_{L}^{\prime} \subset\{[L]\}$ since it is the composition of continuous functions there. To check continuity at $[L]$ note that if $\left\{x_{n}\right\}$ is a sequence in $N_{L}^{\prime}$ converging to $[L]$, then $\left\{s\left(x_{n}\right)\right\}$ is a sequence which is eventually constant and equal to $[L] \in N_{L}$.

Theorem 4.3. Suppose $P=(N, L)$ and $P^{\prime}=\left(N^{\prime}, L^{\prime}\right)$ are filtration pairs for $S$. Then the induced maps, $f_{P}$ and $f_{P^{\prime}}$, on the corresponding pointed spaces, are shift equivalent.

Proof. By Proposition 3.4 we may choose $\varepsilon>0$ sufficiently small that

$$
C_{\varepsilon}(N \backslash L, S)=C_{\varepsilon}\left(N^{\prime} \backslash L^{\prime}, S\right) \subset \operatorname{Int}(N \backslash L) \cap \operatorname{Int}\left(N^{\prime} \backslash L^{\prime}\right) .
$$


Denote $\operatorname{cl}\left(C_{\varepsilon}(N \backslash L, S)\right)$ by $B$. We will show that if $B_{0}$ is a sufficiently small compact neighborhood in $B$ of the exit set $B^{-}$, then $P_{0}=\left(B, B_{0}\right)$ is a filtration pair with the property that $f_{P_{0}}$ is shift equivalent to both $f_{P}$ and $f_{P^{\prime}}$. The argument is the same in both cases so we will give the proof for $f_{P}$.

As before we will identify $N \backslash L$ with $N_{L} \backslash\{[L]\}$ and hence we may consider $B$ as a subset of $N_{L}$ and consider the map to be $f_{P}$. Recall that $B^{-}$is the set of $x \in B=C_{\varepsilon}(N, S)$ such that $f_{P}(x) \notin \operatorname{Int} B$. Thus from the definition of $C_{\varepsilon}(N, S)$ it is clear that $x \in B^{-}$implies there is no $\varepsilon$-chain from $f_{P}(x)$ to $S$. Consequently by Proposition 3.2 the $\omega$-limit set of such an $x$ can only be the point $[L]$. From this it follows that there is an $n>0$ such that for any $x \in B^{-}$we have $f_{P}^{n}(x)=[L]$. If $B_{0}$ is a sufficiently small neighborhood of $B^{-}$in $B$, the same is true for points of $B_{0}$.

Define $K \subset N_{L}$ to be $\operatorname{cl}\left(\operatorname{Int} f_{P}^{-n}([L])\right)$. Then $f_{P}(K) \subset \operatorname{Int} K$ because $f_{P}^{-1}([L])$ is a neighborhood of $[L]$ and any sequence converging to a point of $f_{P}(K)$ will have an image under $f_{P}^{n-1}$ converging to $[L]$. So any such sequence will eventually be in $K$. We also note that $B_{0} \subset \operatorname{Int} K$.

Thus $R=\left(N_{L}, K\right)$ and $Q=(B \cup K, K)$ are filtration pairs for $S$. We complete the proof by showing $f_{R}: N_{L} / K \rightarrow N_{L} / K$ is shift equivalent to both $f_{P}: N_{L} \rightarrow N_{L}$ and $f_{P_{0}}: B_{B_{0}} \rightarrow B_{B_{0}}$. By Lemma $4.1 f_{P_{0}}$ is shift equivalent to $f_{Q}$ and by Lemma 4.2 $f_{Q}$ is shift equivalent to $f_{R}$. Thus $f_{R}$ is shift equivalent to $f_{P_{0}}$

To see that $f_{R}$ is shift equivalent to $f_{P}$ we observe that if $p: N \rightarrow N_{L}$ is the quotient map, then we can identify the associated pointed spaces (and maps) of the filtration pairs $\left(N_{L}, K\right)$ and $\left(N, p^{-1}(K)\right)$. By Lemma 4.1$] f_{P}$ is shift equivalent to the associated pointed space map for the pair $\left(N, p^{-1}(K)\right)$ which we have identified with $f_{R}$.

One could ask if the shift equivalence constructed in Theorem 4.3 is unique. Clearly it is not. For any two spaces and a given lag there may be many different maps $r$ and $s$ giving a shift equivalence. The maps constructed in Theorem 4.3 do have one distinguishing property. Loosely speaking, each map takes a point $x$ either to $f^{k}(x)$ or to the basepoint. We characterize this property as follows.

Definition 4.4. If $r: N_{L} \rightarrow N_{L^{\prime}}^{\prime}$ and $s: N_{L^{\prime}}^{\prime} \rightarrow N_{L}$ define a shift equivalence of lag $m$, we will say that this is a standard shift equivalence provided there exists a non-negative integer $k \leq m$ such that for every $x \in N \backslash L$, either

1. $f^{k}(x) \in N^{\prime} \backslash L^{\prime}$, and $r(x)=p^{\prime}\left(f^{k}(x)\right)$, where $p^{\prime}: N^{\prime} \rightarrow N_{L^{\prime}}^{\prime}$ is the quotient map, or

2. $r(x)=\left[L^{\prime}\right]$.

We call $k$ the semi-lag of $r$. Also we require that the map $s$ has the analogous property with semi-lag $m-k$.

Still, there may be many standard shift equivalences between two pointed spaces. But, in Proposition 4.7 we show that for large enough semi-lag there exist canonical choices for each semi-conjugacy.

Lemma 4.5. The maps $r$ and s defined in Theorem 4.3 give a standard shift equivalence.

Proof. It is immediate from the proofs of Lemmas 4.1 and 4.2 that this result holds in the two special cases covered by those lemmas. But in the general case proved in Theorem 4.3 the semi-conjugacies constructed were all compositions of semi-conjugacies arising in these two special cases. It is clear from the definition 
that the composition of semi-conjugacies of standard shift equivalences is again standard, so the result follows.

Lemma 4.6. Suppose $r$ and $s$ define a standard shift equivalence of lag $m$ from $f_{P}: N_{L} \rightarrow N_{L}$ to $f_{P^{\prime}}: N_{L^{\prime}}^{\prime} \rightarrow N_{L^{\prime}}^{\prime}$ with semi-lags $k$ and $m-k$ respectively. Then if $r^{\prime}$ and $s^{\prime}$ define another standard shift equivalence between $f_{P}$ and $f_{P^{\prime}}$ with the same semi-lags,

$$
r \circ f_{P}^{m}=r^{\prime} \circ f_{P}^{m} \quad \text { and } \quad s \circ f_{P^{\prime}}^{m}=s^{\prime} \circ f_{P^{\prime}}^{m} .
$$

Proof. Let $x \in N_{L}$. If $f_{P}^{m}(x)=[L]$, then clearly $r \circ f_{P}^{m}(x)=r^{\prime} \circ f_{P}^{m}(x)$. On the other hand, if $f_{P}^{m}(x) \neq[L]$, then $r(x) \neq\left[L^{\prime}\right]$ since this would imply $f_{P}^{m}(x)=$ $s(r(x))=s\left(\left[L^{\prime}\right]\right)=[L]$. Likewise we have $r^{\prime}(x) \neq\left[L^{\prime}\right]$. Hence it follows that $r(x)=p^{\prime}\left(f^{k}(x)\right)=r^{\prime}(x)$. From this and the fact that $r$ and $r^{\prime}$ are semi-conjugacies, it is immediate that $r\left(f_{P}^{m}(x)\right)=r^{\prime}\left(f_{P}^{m}(x)\right)$.

The proof that $s \circ f_{P^{\prime}}^{m}=s^{\prime} \circ f_{P^{\prime}}^{m}$ is similar.

The following result is similar to Theorem 6.3 of [RS] in which an analogous result was proved for invertible dynamical systems.

Proposition 4.7. For any two filtration pairs $P=(N, L)$ and $P^{\prime}=\left(N^{\prime}, L^{\prime}\right)$ there exists a number $M\left(P, P^{\prime}\right) \geq 0$ and maps $r_{P^{\prime} P}^{k}: N_{L} \rightarrow N_{L^{\prime}}^{\prime}$ defined for $k \geq M\left(P, P^{\prime}\right)$, with the following properties:

1. $M\left(P, P^{\prime}\right)=M\left(P^{\prime}, P\right)$ and

2. $r_{P P}^{k}=f_{P}^{k}$.

If $P^{\prime \prime}=\left(N^{\prime \prime}, L^{\prime \prime}\right)$ is another filtration pair, then

3. $M\left(P, P^{\prime \prime}\right) \leq M\left(P, P^{\prime}\right)+M\left(P^{\prime}, P^{\prime \prime}\right)$ and

4. $r_{P^{\prime \prime} P}^{k_{1}+k_{2}}=r_{P^{\prime \prime} P^{\prime}}^{k_{1}} \circ r_{P^{\prime} P}^{k_{2}}$.

Proof. By Lemma 4.5 we know that $f_{P}: N_{L} \rightarrow N_{L}$ and $f_{P}^{\prime}: N_{L^{\prime}}^{\prime} \rightarrow N_{L^{\prime}}^{\prime}$ are shift equivalent via a standard shift equivalence. Let $m$ be the minimal lag of all standard shift equivalences. Take $M\left(P, P^{\prime}\right)=2 m$. Defining $M\left(P, P^{\prime}\right)$ in such a way it is clear that properties (1) and (3) hold.

Let $k_{1} \geq M\left(P, P^{\prime}\right)$. We construct $r_{P^{\prime} P}^{k_{1}}: N_{L} \rightarrow N_{L^{\prime}}^{\prime}$ as follows. Let $r: N_{L} \rightarrow N_{L^{\prime}}^{\prime}$ and $s: N_{L^{\prime}}^{\prime} \rightarrow N_{L}$ be a standard shift equivalence realizing the minimal lag $m$ and having semi-lags $k$ and $m-k$ respectively. Define

$$
r_{P^{\prime} P}^{k_{1}}=r \circ f_{P}^{k_{1}-k}
$$

First, notice that since $k_{1}-k \geq m$ Lemma 4.6 implies that $r_{P^{\prime} P}^{k_{1}}$ is independent of $r$ and $s$. Also, if $P=P^{\prime}$, then $M(P, P)=0$ and taking $r=i d$ we see that (2) is satisfied.

Let $k_{2} \geq M\left(P^{\prime}, P^{\prime \prime}\right)$ be given. Let $r^{\prime \prime}: N_{L} \rightarrow N_{L^{\prime \prime}}^{\prime \prime}$ and $s^{\prime \prime}: N_{L^{\prime \prime}}^{\prime \prime} \rightarrow N_{L}$ form a standard shift equivalence with semi-lags $k^{\prime \prime}$ and $m^{\prime \prime}-k^{\prime \prime}$ respectively achieving minimal lag $m^{\prime \prime}$. Observe that $r^{\prime} \circ r$ and $s \circ s^{\prime}$ form a standard shift equivalence between $f_{P}$ and $f_{P^{\prime \prime}}$ with semi-lags $k+k^{\prime}$ and $m+m^{\prime}-k-k^{\prime}$ respectively. Since $k_{1}+k_{2} \geq M\left(P, P^{\prime \prime}\right)=2 m^{\prime \prime}$, it follows that $k_{1}+k_{2}-k^{\prime \prime} \geq m^{\prime \prime}$, the lag of the shift equivalence given by $r^{\prime \prime}$ and $s^{\prime \prime}$. Similarly, $k_{1}+k_{2}-k-k^{\prime} \geq m+m^{\prime}$, the lag of 
$r^{\prime} \circ r$ and $s \circ s^{\prime}$. Thus, by Lemma 4.6 we have

$$
\begin{aligned}
r_{P^{\prime \prime} P}^{k_{1}+k_{2}} & =r^{\prime \prime} \circ f_{P}^{k_{1}+k_{2}-k^{\prime \prime}} \\
& =r^{\prime} \circ r \circ f_{P}^{k_{1}+k_{2}-k-k^{\prime}} \\
& =r^{\prime} \circ f_{P^{\prime}}^{k_{2}-k^{\prime}} \circ r \circ f_{P}^{k_{1}-k} \\
& =r_{P^{\prime \prime} P^{\prime}}^{k_{2}} \circ r_{P^{\prime} P}^{k_{1}} .
\end{aligned}
$$

Thus property (4) holds.

Clearly, if $k, l \geq M\left(P, P^{\prime}\right)$, the above proposition shows that $r=r_{P^{\prime} P}^{k}$ and $s=r_{P P^{\prime}}^{l}$ form a standard shift equivalence between $f_{P}$ and $f_{P^{\prime}}$ of lag $k+l$.

Suppose $S$ is an isolated invariant set. By Theorem 4.3 we know that for any filtration pair $P=(N, L)$ the shift equivalence class of $f_{P}: N_{L} \rightarrow N_{L}$ is an invariant for $S$. This invariant is, however, too restrictive for our desires. Instead consider the homotopy class of base point preserving maps on $N_{L}$ with $f_{P}$ as a representative. We denote this collection $h_{P}(S)$.

Suppose $X$ and $Y$ are pointed topological spaces and $[f]: X \rightarrow X$ and $[g]$ : $Y \rightarrow Y$ are homotopy classes of base-point preserving maps. Then the definition of shift equivalence states that $(X,[f])$ and $(Y,[g])$ are shift equivalent if there exist homotopy classes of maps $[r]: X \rightarrow Y$ and $[s]: Y \rightarrow X$ such that $[g \circ r]=[r \circ f]$, $[s \circ g]=[f \circ s],[r \circ s]=\left[g^{m}\right]$ and $[s \circ r]=\left[f^{m}\right]$ for some $m$.

We may now make the following definition.

Definition 4.8. Let $S$ be an isolated invariant set for a continuous map $f$. Then define the discrete homotopy Conley index of $S, h(S)$, to be the shift equivalence class of $h_{P}(S)$ where $P=(N, L)$ is a filtration pair for $S$.

Once this invariant has been defined, we may apply functors to it to obtain new invariants. For instance, we may take homology to obtain the homological Conley index, $\operatorname{Con}_{*}(S)$. In other words, $\operatorname{Con}_{*}(S)$ is the shift equivalence class of $\left(f_{P}\right)_{*}: H_{*}\left(N_{L},[L]\right) \rightarrow H_{*}\left(N_{L},[L]\right)$ where $P=(N, L)$ is a filtration pair for $S$. Similarly, we define the cohomological Conley index, $\operatorname{Con}^{*}(S)$.

For some situations we may want the Conley index to be a graded abelian group together with a distinguished automorphism. Thus, we may apply a functor to the shift equivalence class $\mathrm{Con}_{*}(S)$ or $\mathrm{Con}^{*}(S)$ such as the Leray functor. One should consult $[\mathrm{Mr}]$ for details.

The Conley index has several important properties.

Theorem 4.9 (Continuation property). Suppose $f_{\lambda}: X \times[0,1] \rightarrow X$ is a continuous homotopy and $N$ is an isolating neighborhood for $f_{0}$ with $S_{0}=\operatorname{Inv}\left(N, f_{0}\right)$. Then there is an $\varepsilon>0$ such that $N$ is an isolating neighborhood for every $f_{\lambda}, \lambda<\varepsilon$. Moreover, $h\left(S_{0}, f_{0}\right)=h\left(S_{\lambda}, f_{\lambda}\right)$ where $S_{\lambda}=\operatorname{Inv}\left(N, f_{\lambda}\right)$ and $\lambda<\varepsilon$.

Proof. This result is an easy consequence of Theorem 3.6

Moreover, if our space is actually a manifold, we have the following stronger version of Theorem 4.9

Corollary 4.10. Let $f: M \rightarrow M$ be a continuous map of a manifold with isolating neighborhood $N$ and isolated invariant set $S=\operatorname{Inv} N$. There is a neighborhood of $f$ in the $C_{0}$ topology with the property that $N$ is an isolating neighborhood for every $\tilde{f}$ in this neighborhood. Moreover, $h(S, f)=h(\tilde{S}, \tilde{f})$ where $\tilde{S}=\operatorname{Inv}(N, \tilde{f})$. 
Proof. We use the fact that we may calculate $h(S, f)$ using a filtration pair homeomorphic to a finite simplicial complex. This implies that for any $\tilde{f}$ sufficiently close to $f$ the maps $f$ and $\tilde{f}$ are homotopic by a homotopy through maps close to $f$.

The corollary given above does not hold for arbitrary locally compact metric spaces. For instance, one may take $X=\left\{\frac{1}{n}: n \in \mathbb{Z}^{+}\right\} \cup\{0\}, f=i d, N=X$ and $L=\emptyset$. Then, for any $\varepsilon>0$ define a map $f_{\varepsilon}: X \rightarrow X$ by putting $f_{\varepsilon}(x)=x$ when $x \geq \varepsilon$ and $f_{\varepsilon}(x)=0$ otherwise. It is clear that the maps $f$ and $f_{\varepsilon}$ are not shift equivalent.

We say that $h(S)=0$ if each pointed space map for $S$ is shift equivalent (in the homotopy category) to the map on the pointed space consisting of one point.

Theorem 4.11 (Ważewski property). The set $\emptyset$ is an isolated invariant set with $h(\emptyset)=0$. In particular, the contrapositive states that if $h(S) \neq 0$, then $S \neq \emptyset$.

Proof. This result follows immediately from Definitions 3.1 and 4.8

\section{Attractors}

Recall that for any $x \in X$ we define the $\omega$-limit set of $x$ to be

$$
\omega(x)=\bigcap_{N>0} \operatorname{cl}\left(\bigcup_{n>N}\left\{f^{n}(x)\right\}\right) .
$$

We would like to do the same for backward iterates. But, because there is no unique backward trajectory of a point, we must modify the definition; we must speak of the backward limit of a solution. For any solution $\sigma: \mathbb{Z}^{-} \rightarrow X$ define

$$
\alpha(\sigma)=\bigcap_{N>0} \operatorname{cl}\left(\bigcup_{n>N}\{\sigma(-n)\}\right) .
$$

A set $A \subset X$ is called an attractor if there exists a compact neighborhood $U$ of $A$ such that

$$
f(U) \subset \operatorname{Int} U
$$

and

$$
A=\bigcap_{n>0} f^{n}(U)
$$

The set $U$ is called an attracting neighborhood of $A$. If $X$ is invariant, then define the dual repeller $A^{*}$ to be

$$
A^{*}=\{x \in X: \omega(x) \cap A=\emptyset\} .
$$

If $X$ is not invariant, then we only consider $x \in \operatorname{Inv} X$. The pair $\left(A, A^{*}\right)$ is called an attractor-repeller decomposition of $X$. For any two sets $B, C \in X$ we define $C(B, C ; X)$, the connecting orbits from $B$ to $C$, to be

$$
\{x \in X \backslash(B \cup C): \exists \text { solution } \sigma: \mathbb{Z} \rightarrow X \text { with } \alpha(\sigma) \subset B, \omega(x) \subset C\} .
$$

Proposition 5.1. If $\left(A, A^{*}\right)$ is an attractor-repeller decomposition of a compact invariant space $X$, then

1. $X=A \cup A^{*} \cup C\left(A^{*}, A ; X\right)$,

2. there exists $\varepsilon>0$ such that there exists no $\varepsilon$-chain from $A$ to $A^{*}$, and

3. A and $A^{*}$ are disjoint isolated invariant sets. 
Proof. The first of these properties is immediate from the definitions. The second follows from the fact that if $U$ is an attracting neighborhood for $A$ and $\varepsilon>d(f(U), \operatorname{cl}(X \backslash U))$, then there exists no $\varepsilon$-chain from a point of $f(U)$ to a point of $\operatorname{cl}(X \backslash U)$. The only non-trivial part of the third property is that $A^{*}$ is an isolated invariant set. This holds because whenever $U$ is an attracting neighborhood for $A$, then $\operatorname{cl}(X \backslash U)$ is an isolating neighborhood for $A^{*}$.

Lemma 5.2. If $V$ and $W$ are attracting neighborhoods, then $V \cap W, V \cup W$ and $f^{-1}(V)$ are attracting neighborhoods.

Proof. These results are immediate except for the fact that $f^{-1}(V)$ is an attracting neighborhood. To see this observe that since $f(V) \subset \operatorname{Int} V$,

$$
f\left(f^{-1}(V)\right) \subset V \subset f^{-1}(f(V)) \subset f^{-1}(\operatorname{Int} V) \subset \operatorname{Int} f^{-1}(V) .
$$

The following lemma is immediate if $f$ is a homeomorphism, but it requires proof otherwise.

Lemma 5.3. Suppose $A$ is an attractor and $V$ is a neighborhood of $A$ in some manifold. Then there is an attracting neighborhood $U \subset V$.

Proof. It suffices to prove the result for an attractor $A$ consisting of a single point since we can always consider the map induced by $f$ on the quotient space $X / A$ which has the single point $[A]$ as attractor.

Thus we assume $A$ consists of one point. Let $N$ be an attracting neighborhood for $A$, so $P=(N, L)$ with $L=\emptyset$ is a filtration pair for the invariant set $A$. The associated pointed space $N_{L}=N \cup[L]$ consists of $N$ together with a disjoint single point $[L]$. The map $f_{P}: N_{L} \rightarrow N_{L}$ has precisely two fixed points, $A$ and $[L]$.

Given any neighborhood $V$ of $A$ it follows from Theorem 3.7 that there is filtration pair $Q=(Y, K)$ for $A$ inside $V$. Let $r: Y_{K} \rightarrow N_{L}$ be one of the semi-conjugacies from the shift equivalence between $f_{Q}$ and $f_{P}$. Then $U_{0}=r^{-1}\left(N_{L}\{[L]\}\right)$ is an open and closed subset of $Y_{K}$ which is forward invariant, contains $A$, and is disjoint from $K$. It follows that $f_{Q}\left(U_{0}\right) \subset$ Int $U_{0}$ so if $p: Y \rightarrow Y_{K}$ is the quotient map, then $U=p^{-1}\left(U_{0}\right)$ is an attracting neighborhood for $A$ which is contained in $V$.

We would like to speak of an attractor-repeller decomposition of an isolated invariant set $S$. Clearly, all the definitions and results given above apply. However, we would like to define our attracting neighborhood as a subset of the ambient space $X$, not as a subset of $S$. An obvious definition of such an attracting neighborhood $U$ would be that $U$ be a neighborhood of $A$ in $X$ with $f(U \cap S) \subset \operatorname{Int} U \cap S$ and $A=\bigcap_{n>0} f^{n}(U \cap S)$. In fact, as we shall see later, given a filtration pair $P=(N, L)$ for $S$, we may choose $U \subset N$ so that $U$ is a neighborhood of $L$ and in the pointed space $N_{L}, f_{P}(U) \subset \operatorname{Int} U$.

\section{MoRse DECOMPOSITIONS}

Suppose $\mathcal{P}$ is a finite set. We say that a relation $<$ is a partial order on $\mathcal{P}$ provided that for all $p, q \in \mathcal{P}$ :

1. $p<p$ never holds, and

2. $p<q$ and $q<r$ implies $p<r$.

3. If, in addition, for any $p \neq q$ we have $p<q$ or $q<p$, then $<$ is called a total ordering. 
Suppose $\mathcal{P}$ has a partial order $<$. A subset $I \subset \mathcal{P}$ is called an interval if $p<r<q$ and $p, q \in I$, then $r \in I$. An interval $I$ is called attracting provided $r<q$ and $q \in I$ implies $r \in I$. We will denote the attracting interval with unique maximal element $p$ by $I_{p}$. We denote the collection of intervals and attracting intervals by $\mathcal{I}=\mathcal{I}(\mathcal{P},<)$ and $\mathcal{A}=\mathcal{A}(\mathcal{P},<)$ respectively.

Definition 6.1. The collection of disjoint isolated invariant sets $\mathcal{M}(\mathcal{P},<)=$ $\left\{M_{p} \subset S: p \in \mathcal{P}\right\}$ is a Morse decomposition of an isolated invariant set $S$ if for every $x \in S$ and every solution $\sigma: \mathbb{Z} \rightarrow S$ for $x$ we have either

1. $\sigma(\mathbb{Z}) \subset M_{p}$ for some $p \in \mathcal{P}$, or

2. $\omega(\sigma) \subset M_{p}$ and $\alpha(\sigma) \subset M_{q}$ for some $p<q$.

For any interval $I \subset \mathcal{P}$ define the set

$$
M_{I}=\bigcup_{p \in I} M_{p} \cup \bigcup_{q, r \in I} C\left(M_{q}, M_{r} ; S\right) .
$$

Notice that the partial ordering on the Morse sets is analogous to Smale's nocycle property for basic sets of an Axiom A diffeomorphism [S].

Proposition 6.2. Suppose $f(X) \subset \operatorname{Int} X$ so $S=\operatorname{Inv} X$ is an attractor in $X$. Let $\mathcal{M}(\mathcal{P},<)=\left\{M_{p} \subset S: p \in \mathcal{P}\right\}$ be a Morse decomposition of $S$. If $I \subset \mathcal{P}$ is an attracting interval, then $M_{I}$ is an attractor, i.e. there is a compact neighborhood $U_{I} \subset X$ of $M_{I}$ such that

1. $f\left(U_{I}\right) \subset \operatorname{Int} U_{I}$, and

2. $M_{I}=\operatorname{Inv}\left(U_{I}\right)=\bigcap_{n>0} f^{n}\left(U_{I}\right)$.

Moreover, if $V_{I}$ is an open neighborhood of $M_{I}$, then we can find $U_{I} \subset V_{I}$.

Proof. An attracting interval $I$ in $\mathcal{P}$ for the order $<$ is also an attracting interval for a total ordering of the set $\mathcal{P}$ which is compatible with $<$. Thus, without loss of generality we may assume that $<$ is a total order.

We will give the proof by induction on the number of elements $n$ in $\mathcal{P}$. Clearly if $n=1$, then $M_{I}=S$ so we may take $U_{I}=X$. Hence we may assume the result for $n=r-1$ and need to prove it for $n=r$.

Let $p_{r}$ be the maximal element of $\mathcal{P}$ and define $J$ to be the attracting interval $\mathcal{P} \backslash\left\{p_{r}\right\}$. Let $B=\bigcup_{p \in J} M_{p}$ and choose a compact neighborhood $V$ of $B$ which is disjoint from $M_{p_{r}}$. We wish to show that for some smaller neighborhood $W$ of $B$ we have the property that $\omega(x) \subset B$ for all $x \in W$. We assume this is not the case and show that it leads to a contradiction.

If no such $W$ exists, then there is a convergent sequence $\left\{x_{n}\right\}$ such that $\lim x_{n} \in$ $B$ and $\omega\left(x_{n}\right)$ is not a subset of $B$. From this it follows that we can choose $y_{n}$ with the properties:

1. $y_{n} \in V$,

2. $f^{k}\left(y_{n}\right) \notin V$ for $0<k<n$, and

3. $y_{n}=f^{j_{n}}\left(x_{n}\right)$ for some $j_{n}>0$.

By choosing a subsequence we may assume that the sequence $\left\{y_{n}\right\}$ converges, say to $z \in V$. We claim that $z \in S$. This follows because $f^{k}(z) \in X$ for all $k>0$ and for any $m>0$ the sequence $\left\{w_{n}=f^{j_{n}-m}\left(x_{n}\right)\right\}$ is well defined for sufficiently large $n$ and has a subsequence which converges to a point of $f^{-m}(z)$. Thus we have shown $z \in \operatorname{Inv}^{m} X$ for all $m>0$ so by Lemma 2.2 we have $z \in \operatorname{Inv} X=S$.

Also, for every $k>0 f^{k}(z) \notin \operatorname{Int} V$ since $f^{k}(z)=\lim _{n \rightarrow \infty} f^{k}\left(y_{n}\right)$. Thus $\omega(z)$ is disjoint from Int $V$ and the definition of Morse decomposition implies that $\omega(z) \subset$ 
$\mathcal{M}_{p_{r}}$. Let $\sigma$ be a solution for $z$. Then $\alpha(z) \subset \mathcal{M}_{q}$ for some $q \in \mathcal{P}$, so $q \geq p_{r}$. But this leads to a contradiction since $p_{r}$ is maximal, so $q>p_{r}$ is impossible, but $q=p_{r}$ would imply that $z \in M_{p_{r}}$ which is false.

Thus we have shown that there is a compact neighborhood $W \subset V$ of $B$ with the property that $\omega(x) \subset B$ for all $x \in W$. To each $x \in W$ we may associate $n(x)$ defined to be the smallest non-negative integer such that $f^{n(x)}(x) \in \operatorname{Int} W$. The function $n: W \rightarrow \mathbb{Z}^{+}$is upper semicontinuous. Since $W$ is compact $N=$ $\max \{n(x): x \in W\}$ is finite. Let $U_{J}=f^{-N}(W)$. If $x \in U_{J}$, then $f^{k}(x) \in \operatorname{Int} W$ for some $0<k \leq N$ so $f(x) \in f^{-N}(\operatorname{Int} W) \subset \operatorname{Int} U_{J}$. Hence $f\left(U_{J}\right) \subset \operatorname{Int} U_{J}$. Since $M_{p_{r}} \cap U_{J}=\emptyset$ and $M_{J} \subset U_{J}$, it follows that $M_{J}=\operatorname{Inv} U_{J}$. The fact that $\operatorname{Inv} U_{J}=\bigcap_{n>0} f^{n}\left(U_{J}\right)$ follows from Lemma 2.2.

If $I=J$, then we're done. If $I \neq J$, then $I$ is a subinterval of $J$. Note that $\mathcal{M}(J,<)$ is a Morse decomposition for the map $f: U_{J} \rightarrow U_{J}$ with attractor $M_{J}$. Since $J$ has one fewer element than $\mathcal{P}$, our induction hypothesis implies there is an attracting neighborhood $U_{I} \subset U_{J}$ for $M_{I}$.

Finally, we remark that by Lemma 5.3 we may assume that $U_{I} \subset V_{I}$.

Suppose $\mathcal{M}(\mathcal{P},<)=\left\{M_{p} \subset S: p \in \mathcal{P}\right\}$ is a Morse decomposition of an isolated invariant set $S$, and $P=(N, L)$ is a filtration pair with associated pointed space map $f_{P}: N_{L} \rightarrow N_{L}$. Then $N_{L}$ is an attracting neighborhood for an attractor $\hat{S}=$ Inv $N_{L}=\bigcap_{n>0} f_{P}^{n}\left(N_{L}\right)$. Moreover there is a Morse decomposition of $\hat{S}$ associated to $\mathcal{M}(\mathcal{P},<)$ obtained by adding the single point $[L]$ as a minimal Morse set. More precisely, we define the associated Morse decomposition as follows.

Definition 6.3. Let $\hat{\mathcal{P}}=\mathcal{P} \cup\{0\}$ and specify $0<p$ for all $p \in \mathcal{P}$. Define the associated Morse decomposition to $\mathcal{M}(\mathcal{P},<)$ to be

$$
\hat{\mathcal{M}}(\mathcal{P},<)=\left\{\hat{M}_{p}: \hat{M}_{p}=M_{p}, p \in \mathcal{P}\right\} \cup\left\{M_{0}\right\},
$$

where $M_{0}=\{[L]\}$.

It is not immediately clear that the associated Morse decomposition is in fact a Morse decomposition, so we prove that now.

Proposition 6.4. Suppose $\mathcal{M}(\mathcal{P},<)$ is a Morse decomposition for the isolated invariant set $S$ with filtration pair $(N, L)$. Then the associated Morse decomposition $\hat{\mathcal{M}}(\hat{\mathcal{P}},<)$ is a Morse decomposition for the attractor $\hat{S}=\operatorname{Inv} N_{L}$.

Proof. The sets $\hat{M}_{p}$ with $p \in \hat{\mathcal{P}}$ can immediately be seen to satisfy the properties of the definition of Morse decomposition 6.1 with one exception. If $z \in \hat{S}$ with $\omega(z)=\{[L]\}$ and $\sigma$ is a solution for $z$, then it is not immediate that $\alpha(\sigma) \subset \hat{M}_{q}$ for some $q \in \hat{\mathcal{P}}$. However, the proof of Proposition 6.2 did not make use of this property so the conclusion of this proposition is valid for $\hat{\mathcal{M}}(\hat{\mathcal{P}},<)$. In particular if $I \subset \mathcal{P}$ is an attracting interval, then $M_{I}$ is an attractor.

We now consider $z \in \hat{S}$ with $\omega(z)=\{[L]\}$ and let $\sigma$ be a solution for $z$. Since $\alpha(\sigma) \subset S$ and $S$ has a Morse decomposition given by $\left\{\hat{M}_{p}: p \neq 0\right\}$ it must be the case that either $\alpha(\sigma)$ is a subset of a single $\hat{M}_{q}$ or $\alpha(\sigma)$ contains points of $\hat{M}_{q}$ and $\hat{M}_{p}$ with $p \neq q$. But if the attracting interval $I$ is chosen to contain one of $p$ and $q$ and not the other, then the existence of the attracting neighborhood $U_{I}$ makes it impossible for $\alpha(\sigma)$ to contain points of both $\hat{M}_{q}$ and $\hat{M}_{p}$. Thus $\alpha(\sigma) \subset \hat{M}_{q}$ for some $q \in \mathcal{P}$. 
Corollary 6.5. If $\mathcal{M}(\mathcal{P},<)$ is a Morse decomposition of an isolated invariant set $S$, then for any interval $I \subset \mathcal{P}$ the set $M_{I}$ is an isolated invariant set.

Proof. Without loss of generality we may assume that the hypotheses of Proposition 6.2 hold, if not, then by Proposition 6.4 we may simply consider the pointed space for $S$ and the associated Morse decomposition there. Moreover, since any interval is also an interval for a total ordering we may assume $(\mathcal{P},<)$ is a total order. In this order there are attracting intervals $J_{0}$ and $J_{1}$ such that $J_{1}=J_{0} \cup I$. Consider the attracting neighborhood $U_{1}$ for $\mathcal{M}_{J_{1}}$ given by Proposition6.2 and the map $f: U_{1} \rightarrow U_{1}$. A Morse decomposition is given by $\mathcal{M}\left(J_{1},<\right)$. Another application of Proposition 6.2 implies that $\mathcal{M}_{J_{0}}$ is an attractor with attracting neighborhood $U_{0} \subset U_{1}$. Clearly, with respect to $f: U_{1} \rightarrow U_{1}$ the set $M_{I}$ is the dual repeller to $M_{J_{0}}$ and hence is closed. The set $N=U_{1} \backslash \operatorname{Int} U_{0}$ is an isolating neighborhood of $M_{I}$.

\section{Morse Set fiLtrations}

A variation of the following definition was introduced by Franzosa to study Morse decompositions for continuous time dynamical systems $[\mathrm{F}$. It is also similar to the neighborhood networks introduced by Robbin and Salamon [RS] for discrete dynamical systems.

Definition 7.1. A collection of compact sets $\mathcal{N}(\mathcal{P},<)=\{N(I) \subset X: I \in \mathcal{A}\}$ is called a Morse set filtration for $\mathcal{M}(\mathcal{P},<)$ provided that for any attracting intervals $I, J \subset \mathcal{P}$ the following conditions hold.

1. $(N(I), N(\emptyset))$ is a filtration pair for $M_{I}$,

2. $N(I) \cap N(J)=N(I \cap J)$, and

3. $N(I) \cup N(J)=N(I \cup J)$.

Proposition 7.2. Suppose $\mathcal{N}(\mathcal{P},<)$ is a Morse set filtration for a Morse decomposition $\mathcal{M}(\mathcal{P},<)$ and $J \subset I$ are attracting intervals. Then $(N(I), N(J))$ is a filtration pair for $M_{I \backslash J}$.

Proof. From property (2) of the definition above it is clear that $N(I) \subset N(J)$ whenever $I$ and $J$ are attracting intervals with $J \subset I$. It is then straightforward to check that $(N(I), N(J))$ satisfies the requirements of Definition 3.1

Lemma 7.3. Suppose $f(X) \subset \operatorname{Int} X$ and $S=\operatorname{Inv} X$ has a Morse decomposition $\mathcal{M}(\mathcal{P},<)=\left\{M_{p} \subset S: p \in \mathcal{P}\right\}$. Then there exists a collection

$$
\left\{V(I): V(I) \text { is an attracting neighborhood for } M_{I}, I \in \mathcal{A}\right\}
$$

with the property that $V(I) \cap V(J)=V(I \cap J)$ and $V(I) \cup V(J)=V(I \cup J)$.

Proof. We shall build the $V(I)$ inductively. The induction hypothesis is as follows. Suppose $J \subset \mathcal{P}$ is an attracting interval and that for each attracting interval $I \subset J$ there is an attracting neighborhood $V(I)$ satisfying the conclusions of the lemma. Moreover, if $p \in J$ and $q \notin J$ with $p \nless q$, then $\operatorname{cl}\left(V\left(I_{p}\right) \backslash V\left(I_{p} \cap I_{q}\right)\right) \cap M_{I_{q}}=\emptyset$.

For $J=\emptyset$ take $V(\emptyset)=\emptyset$. Suppose the induction holds for an attracting interval $J$ and $q$ is minimal in $\mathcal{P} \backslash J$. We will define $V(I)$ for all $I$ in the attracting interval $J \cup\{q\}$. Without loss of generality we may assume that for each $p \neq q$ we have $M_{I_{p}} \cap M_{I_{q}} \subset V\left(I_{p} \cap I_{q}\right)$. If not, then we may replace each $V(I)$ by $f^{-N}(V(I))$ for some $N$ sufficiently large. 
By the induction hypothesis and the above remark, there exists an open neighborhood $U$ of $M_{I_{q}}$ such that $U$ is disjoint from $\operatorname{cl}\left(V\left(I_{p}\right) \backslash V\left(I_{p} \cap I_{q}\right)\right)$ for all $p \in J$, $p \nless q$ and that $U$ is disjoint from $\operatorname{cl}\left(M_{I_{p}} \backslash V\left(I_{p} \cap I_{q}\right)\right)$ for all $p \nless q$. By Lemma 5.3 there is an attracting neighborhood $V \subset U$ for $M_{I_{q}}$. Define

$$
V\left(I_{q}\right)=V \cup\left(\bigcup_{p \in I_{q} \backslash\{q\}} V\left(I_{p}\right)\right)
$$

and more generally for any attracting interval $I \subset J \cup\{q\}$

$$
V(I)=\bigcup_{p \in I} V\left(I_{p}\right)
$$

It is straightforward to check that this construction completes the inductive step.

Theorem 7.4. Suppose $\mathcal{M}(\mathcal{P},>)$ is a Morse decomposition of an isolated invariant set $S$. Then there exists a Morse set filtration for $\mathcal{M}(\mathcal{P},<)$.

Proof. Let $P=(N, L)$ be a filtration pair for $S$ and $\hat{S}=\operatorname{Inv} N_{L}$. Let $\hat{\mathcal{M}}(\hat{\mathcal{P}},<)$ denote the associated Morse decomposition of $\hat{S}$. By Lemma 7.3 there exists a collection

$$
\left\{V(I) \subset N_{L}: V(I) \text { is an attracting neighborhood for } M_{I}, I \in \mathcal{A}(<)\right\}
$$

with the property that $V(I) \cap V(J)=V(I \cap J)$ and $V(I) \cup V(J)=V(I \cup J)$. To obtain a Morse set filtration for $\mathcal{M}(\mathcal{P},<)$ we simply take $N(I)=q^{-1}(V(I \cup\{0\}))$ where $0 \in \hat{\mathcal{P}}$ corresponds to the Morse set $\{[L]\}$ and $q: N \rightarrow N_{L}$ is the quotient map. It is clear that this collection forms a Morse set filtration.

\section{Shift equivalence and Szymczak's category}

In [Sz] Szymczak provides a different, categorical, definition of the Conley index. We conclude this discussion by showing that his definition is identical to ours.

For any category $\mathcal{K}$ define the category of objects equipped with a morphism over $\mathcal{K}$, denoted $\mathcal{K}_{m}$, as follows.

$$
\begin{gathered}
\operatorname{Ob}\left(\mathcal{K}_{m}\right)=\left\{(X, f): X \in \operatorname{Ob}(\mathcal{K}), f \in \operatorname{Mor}_{\mathcal{K}}(X, X)\right\}, \\
\operatorname{Mor}_{\mathcal{K}_{m}}\left((X, f),\left(X^{\prime}, f^{\prime}\right)\right)=M\left((X, f),\left(X^{\prime}, f^{\prime}\right)\right) / \sim
\end{gathered}
$$

where

$$
M\left((X, f),\left(X^{\prime}, f^{\prime}\right)\right)=\left\{(g, n) \in \operatorname{Mor}_{\mathcal{K}}\left(X, X^{\prime}\right) \times \mathbb{Z}^{+}: g f=f^{\prime} g\right\}
$$

and $\sim$ is the following equivalence relation. $\left(g_{1}, n_{1}\right) \sim\left(g_{2}, n_{2}\right)$ if and only if there exists $k \in \mathbb{Z}^{+}$such that the following diagram commutes.

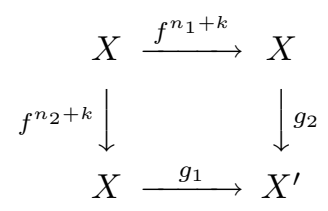

Henceforth we shall call this category the Szymczak category.

Proposition 8.1. Suppose that $(X, f),\left(X^{\prime}, f^{\prime}\right) \in \mathrm{Ob}\left(\mathcal{K}_{m}\right)$. Then $(X, f)$ and $\left(X^{\prime}, f^{\prime}\right)$ are isomorphic in the Szymczak category if and only if they are shift equivalent. 
Proof. Suppose $f$ and $f^{\prime}$ are shift equivalent with $r s=f^{m}$ and $s r=\left(f^{\prime}\right)^{m}$. We claim that $[r, m] \in \operatorname{Mor}_{\mathcal{K}_{m}}\left(\left(X^{\prime}, f^{\prime}\right),(X, f)\right)$ is an isomorphism with inverse $[s, 0] \in$ $\operatorname{Mor}_{\mathcal{K}_{m}}\left((X, f),\left(X^{\prime}, f^{\prime}\right)\right)$. Because the diagram

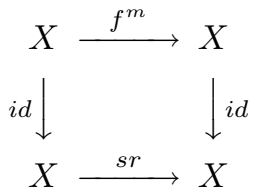

commutes we see that $(s, 0) \circ(r, m)=(s r, m) \sim\left(i d_{X}, 0\right)$. Similarly, $(r s, m) \sim$ $\left(i d_{X^{\prime}}, 0\right)$. Thus, $[r, m]$ and $[s, 0]$ are inverse isomorphisms.

Now suppose that $(X, f)$ and $\left(X^{\prime}, f^{\prime}\right)$ are isomorphic objects in Szymczak's category. In particular, suppose $[r, t] \in \operatorname{Mor}_{\mathcal{K}_{m}}\left((X, f),\left(X^{\prime}, f^{\prime}\right)\right)$ and $[s, u] \in$ $\operatorname{Mor}_{\mathcal{K}_{m}}\left(\left(X^{\prime}, f^{\prime}\right),(X, f)\right)$ are inverse isomorphisms. We have $(s r, t+u) \sim\left(i d_{X}, 0\right)$ and $(r s, t+u) \sim\left(i d_{X^{\prime}}, 0\right)$. Thus, we have $r f=f^{\prime} r, f s=s f^{\prime}$ and $k_{1}, k_{2} \in \mathbb{Z}^{+}$such that the following diagrams commute:
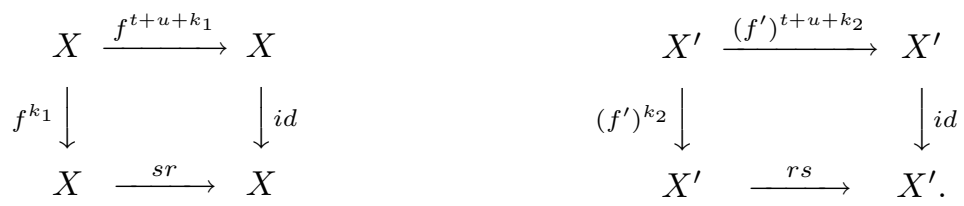

Let $k=\max \left\{k_{1}, k_{2}\right\}, m=t+u+k, s^{\prime}=s$ and $r^{\prime}=r f^{k}$. So defined, we have $f s^{\prime}=s^{\prime} f^{\prime}, r^{\prime} f=f^{\prime} r^{\prime}, r^{\prime} s^{\prime}=\left(f^{\prime}\right)^{m}$ and $s^{\prime} r^{\prime}=f^{m}$. Thus $f$ and $f^{\prime}$ are shift equivalent.

Let Htop * be the category of pointed topological spaces with homotopy classes of base-point preserving maps. An immediate consequence of Proposition 8.1 is that Szymczak's definition of the Conley index is equivalent to ours. We state this explicitly in the following proposition.

Proposition 8.2. Suppose $P=(N, L)$ is a filtration pair for an isolated invariant set $S$. Then the isomorphism class of $\left(N_{L}, f_{P}\right)$ in the Szymczak category $\left(\text { Htop }_{*}\right)_{m}$ is precisely $h(S)$, the Conley index of $S$.

\section{REFERENCES}

[BF] R. Bowen and J. Franks, Homology for zero dimensional non-wandering sets, Annals of Math. 106 (1977), 73-92. MR 56:16692

[C] C. Conley. Isolated invariant sets and the Morse index. CBMS Reg. Conf. Ser. in Math., 38, Amer. Math. Soc., Providence, (1978). MR 80c:58009

[E] Robert Easton, Isolating blocks and epsilon chains for Maps, Physica D 39 (1989) 95-110. MR 90m:58176

[F] R. Franzosa, Index filtrations and the homology index braid for partially ordered Morse decompositions, Trans. Amer. Math. Soc. 298 (1986) 193-213. MR 88a:58121

[F2] R. Franzosa, The connection matrix theory for Morse decompositions, Trans. Amer. Math. Soc. 311 (1989), 561-592. MR 90a:58149

[Mr] M. Mrozek, Leray functor and cohomological Conley index for discrete dynamical systems, Trans. Amer. Math. Soc. 318 (1990), 149-178. MR 90f:34076

[R] D. Richeson, Connection matrix pairs for the discrete Conley index, Ph.D. Dissertation, Northwestern University (1998).

[RS] J. Robbin and D. Salamon, Dynamical systems, shape theory and the Conley index, Ergod. Th. Dyn. Sys. 8 (1988) 375-393. MR 89h:58094 
[RS2] J. Robbin and D. Salamon, Lyapunov maps, simplicial complexes and the Stone Functor, Ergod. Th. Dyn. Sys. 12 (1992), 153-183. MR 93h:58091]

[S] S. Smale, Differentiable dynamical systems. Bull. Amer. Math. Soc., 73 (1967), 747-817. MR 37:3598

[Sz] A. Szymczak, The Conley index for discrete semidynamical systems, Top. App. 66 (1995), 215-240. MR 97f:58113

[W] R. F. Williams, Classification of one dimensional attractors, Global Analysis (Proc. Sympos. Pure Math., Vol XIV, Berkeley, Calif, 1968) (1970), 341-361. MR 42:1134

Department of Mathematics, Northwestern University, Evanston, Illinois 60208-2730

E-mail address: john@math.nwu.edu

Department of Mathematics, Michigan State University, East Lansing, Michigan 48023-1027

E-mail address: richeson@math.msu.edu 